\title{
APPROXIMATION BY SMOOTH EMBEDDED HYPERSURFACES WITH POSITIVE MEAN CURVATURE
}

\author{
Fang Hua Lin
}

\begin{abstract}
Here we initiate the study of the following problem. Let $\Omega$ be a compact domain in a Riemannian manifold such that $\partial \Omega$ is of minimum area for the contained volume. Can $\partial \Omega$ be approximated by smooth hypersurfaces of positive mean curvature? It reduces to the question of whether or not a stable (or minimizing) hypercone in a Euclidian space can be approximated by smooth hypersurfaces of positive mean curvature. The positive solution to the problem may be useful for studying the curvature and topology of $\Omega$

We show in this paper that such approximation is possible provided that the given minimal cone satisfies some additional hypothesis.
\end{abstract}

\section{Introduction}

The aim of this note is to initiate the study of the following problem which was posed by Lawson (see [1, Problems section]). Let $\llbracket$ be a stable (or minimizing) hypercone in $\mathbb{R}^{n+1}$. Given $\varepsilon>0$, can one find a smooth embedded hypersurface $M$ of positive mean curvature in $B_{1}(0) \cap \mathbb{Q}_{\varepsilon}$ so that $\partial M$ is close to $\partial\left(\mathbb{C L} B_{1}(0)\right.$ ) (where $\mathbb{U}_{\varepsilon}$ is the $\varepsilon-$ neighbourhood of $\mathbb{T}$ in $\mathbb{R}^{n+1}$ )? This is related to the following question. Let $\Omega$ be a compact domain in a Riemannian manifold $N$ such that $\partial \Omega$ is of a minimum area for the contained volume. Can $\partial \Omega$ be approximated by a smooth hypersurface of positive mean curvature? The

Received 4 th September 1986. I am very grateful to Professor R. Hardt for various helpful conversations during this work.

Copyright Clearance Centre, Inc. Serial-fee code: 0004-9729/87 $\$ 2.00+0.00$. 
latter is useful for studying the curvature and topoloty of $\Omega$, see for example [6].

Under the additional hypothesis that the given cone $\mathbb{L}$ is regular, that is $\mathbb{L}=0 \# \Sigma$ for some smooth embedded minimal hypersurface of $\Sigma$ of $S^{n}$, both the local and the global approximation problems are studied. In order to make our statements more precise, we fix an orientation on the minimal cone $\mathbb{L}$ so that $B^{n+1}-\mathbb{l}=E_{+} \cup E_{-}$, where $E_{ \pm}$are two connected components of $\mathbb{R}^{n+1} \sim \mathbb{L}$, and $v_{c}$ points into $E_{+} \cdot$ suppose $M$ is a connected, orientable hypersurface and such that $M L\left(B_{1} \sim B_{1 / 2}\right)=$ $\left\{y=x+u(x) v_{c}(x): x \in(\mathbb{C}) L\left(B_{1}-B_{1 / 2}\right)\right.$ is a graph of a small $C^{2}-$ function $u$ over the cone. Then the orientation vector $v_{M}$ of $M$ will be chosen so that $v_{e}(x) v_{M}(y)>0$ for $y=x+u(x) v_{c}(x)$.

Now we can describe our results. In section II, we consider the local approximation problem, and we have

THEOREM 1. Let $\mathbb{E}$ be a regular minimal hypercone in $\mathbb{H}^{n+1}$ with $E_{+}$defined as above. Then, for any integer $k \geq 3$, we have:

(i) if $n \leq 7$, then for every $\varepsilon>0$ there is a smooth properly embedded, connected hypersurface with boundary $M_{\varepsilon}$ supported in $B_{1}(0) \cap \mathbb{C}_{\varepsilon}$ so that $\partial M_{\varepsilon}$ is $c^{k+1}$ close to $\mathbb{L} L \mathbb{S}^{n}$, and $M_{\varepsilon}$ is of positive mean curvature;

(ii) if $\mathbb{L}$ in (i) is, in addition, stable (then $n=7$ is automatic), then $M_{\varepsilon}$ can be chosen to satisfy the additional property that $\operatorname{spt}\left(M_{\varepsilon}\right) \subset B_{1}(0) \cap \mathbb{L}_{\varepsilon} \cap E_{+} ;$

(iii) if $\mathbb{C}$ is one-sided area minimizing in $\bar{E}_{+}$, then, for each $\varepsilon>0$, there are smooth properly embedded, connected hypersurfaces $M^{+}$ and $M_{\varepsilon}$ with positive and negative mean curvature, respectively, in $B_{1}(0) \cap \mathbb{I}_{\varepsilon} \cap E_{+}$, and such that $M_{\varepsilon}^{ \pm}$are $c^{k+1}$ close to $\mathbb{L} L \mathbb{S}^{n}$.

The notion of one-sided area minimality is introduced also in Section II. By a maximum principle (see Lemma 1 in section II) and the regularity of solutions to a parametric obstacle problem [8], we show the equivalence of the existence of $M_{\varepsilon}^{-}$(for suitable small positive $\left.\varepsilon^{\prime} s\right)$ and the one-sided area minimality of $\mathbb{L}$ in $\bar{E}_{+}$. 
In Section III, we study the global approximation problem. There we obtain the following:

THEOREM 2. Let $\mathbb{L}$ and $E_{+}$be as in Theorem 1. We hove:

(i) if $\mathbb{I}$ is not crea-minimizing in $\bar{E}_{+}$, and if $n \leq 7$, then for every $\varepsilon>0$ there is a smooth properly embedded, complete (noncompact) hypersurface $M_{\varepsilon}$ in $\bar{l}_{\varepsilon}$ with positive mean curvature;

(ii) if $\mathbb{L}$ is strictly one-sided area minimizing in $\bar{E}_{+}$, then for each $\varepsilon>0$ there are smooth properly embedded, complete (non-compact) hypersurfaces $M_{\varepsilon}^{+}$and $M_{\varepsilon}$ in $\mathbb{L}_{\varepsilon} \cap E_{+}$with positive and negative meancurvature, respectively.

The strictly one-sided area minimality of a minimal cone and the proof of the existence of $M_{E}$ in the above Theorem are modified from the recent work [ 5]. See also [7] for the related discussions. It still remains as an open problem whether or not every one-sided area minimizing hypercone (not $\mathbb{R}^{2}$ ) is strictly one-sided area minimizing.

In the final section, we show how the results in section II can be generalized. In particular, a similar approximation result is valid for 7-dimensional area minimizing oriented boundaries or minimizing oriented boundaries with a volume constraint. For $n>7$, some additional hypotheses on singular set are needed.

\section{Local approximation}

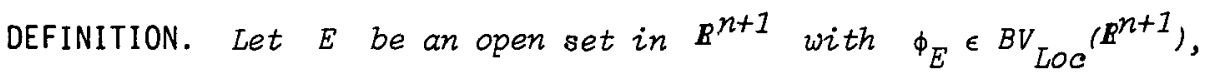
where $\phi_{E}$ is the characteristic function of the set $E$. Let $T=\partial E$ and we say $T$ is area minimizing in $\bar{E}$ (the closure of $E$ ) if the following condition is satisfied: for any compact subset $K$ of $\boldsymbol{B}^{n+1}$, and any $E \subset E$ with $\phi_{F} \in B V_{L o c}\left(\boldsymbol{R}^{n+1}\right)$ and $\overline{E-F}$ a compact subset of $\bar{E}$, one has $\delta_{K}\left|D \phi_{E}\right| \leq \delta_{K}\left|D \phi_{F}\right|$.

It is clear that $\mathbb{C}$ is area minimizing in $\bar{E}_{+}$if and only if $\mathbb{L}_{1}=\mathbb{C} L B_{1}$ is an area minimizing integral current whose support lies in $\bar{E}_{+} \cap B_{1}$ and whose boundary is $\mathbb{E} L \Phi^{n}$. Later we will also say $\mathbb{L}$ is one-sided area minimizing in $\bar{E}_{+}$. By the regularity theorem for the onesided area minimizing currents, see [8], we conclude that $\mathbb{l}_{1}$ is the unique such area minimizing integral current. 
Suppose $\mathbb{C}$ is area minimizing in $\bar{E}_{+}$, then there is a unique area minimizing, smooth properly embedded hypersurface $S_{+}$in $E_{+}$with dist $\left(0, S_{+}\right)=1$, see [5] and [7]. Moreover, $S_{+}$is a polar graph, that is, $x \cdot v_{S_{+}}(x)>0$, for all $x \in \operatorname{spt}\left(S_{+}\right)$; and for some constant $R_{0}=R_{0}(\bar{l})>1$,

$$
S_{+} \sim B_{R_{0}}(0)=\left\{x+u_{+}(x) v_{c}(x): x \in \mathbb{E}\right\} L\left(R^{n+1} \sim B_{R_{0}}(0)\right)
$$

for some $C^{2, \alpha}$ function $u_{+}$on $\mathbb{l}$ with

either $u_{+}=\left(C_{1}+C_{2} \log \gamma\right) \gamma^{-\gamma_{-}} \phi_{1}+o\left(\gamma^{-\gamma_{-}-\alpha}\right)$ as $\gamma \rightarrow \infty$

or $\quad u_{+}=c \gamma^{-\gamma_{+}} \phi_{1}+o\left(\gamma^{-\gamma_{+}^{-\alpha}}\right)$ as $\gamma \rightarrow \infty$

where $C_{1}+c_{2} \log \gamma>0$ in the first identity, and $c_{2}=0$ unless $r_{+}=r_{-}=\frac{n-2}{2}$, and $C>0$ in the second identity. Moreover $\gamma_{+} \geq \gamma_{-}>0$ in our case, see [5].

The following Lemma will be needed in the proof of Theorem $I$.

LEMMA 3. Let $T=\partial E$ be an oriented boundary with $E$ an open subset of $B^{n+1}$. Let spt $T \sim\{0\}$ be a $C^{2}$-hypersurface in $B_{2}$ and spt $(T) \sim\{0\}$ have non-negative mean curvature with respect to the unit normal vector fiezd pointing into $E$. Suppose spt $T$ intersects $\$ n$ transversely, and let $T^{*}$ be con area minimizing integral current whose support lies in $\bar{E}$ and whose boundary is $T L \Phi^{n}$. Then either $T^{*}=T L B_{1}$ or $\operatorname{spt}\left(T^{*}\right) \cap \operatorname{spt} T=\operatorname{spt}\left(T L \Phi^{n}\right)$.

The proof of the above Lemma is contained in the following more general maximum-principle:

MAXIMUM PRINCIPLE. Let $\partial A, \partial B$ be oriented boundaries of open sets $A, B$ and let $x_{0} \in \partial A \cap \partial B$. Suppose that $\partial A, \partial B$ core area-minimizing in $U^{n+1}\left(x_{0}, 2 \rho\right)$, where $0<2 \rho<\min \left\{\operatorname{dist}\left(x_{0}, \partial(\partial A)\right)\right.$, dist $\left.\left(x_{0}, \partial(\partial B)\right)\right\}$. In addition, we assume $A \subset B$. Then $\operatorname{dist}\left(\partial A L \partial B_{\rho}\left(x_{0}, \partial B L \partial B_{\rho}\left(x_{0}\right)\right.\right.$ can not be positive. 
Proof. Suppose $\operatorname{dist}\left(\partial A L \partial B_{\rho}\left(x_{0}\right), \partial B L \partial B_{\rho}\left(x_{0}\right)\right)>0$. By [11] we have regularity of an oriented area minimizing boundary, so we see $\partial A L \partial B_{\rho}\left(x_{0}\right)$ and $\partial B L \partial B_{\rho}\left(x_{0}\right)$ are $(n-1)$-dimensional integral cycles, and the subregion of $S_{\rho}^{n}\left(x_{0}\right)$ which is bounded by $\partial A L \partial B_{\rho}\left(x_{0}\right)$ and $\partial B L \partial B_{\rho}\left(x_{0}\right)$ is well-defined. Now we can choose $(n-1)$-dimensional integral cycles which are supported strictly in the above subregion and which are arbitrary close to $\partial A L \partial B_{\rho}\left(x_{0}\right)$ in flat norm. Let $\Gamma$ be such an integral cycle, we solve the oriented plateau problem with boundary $\Gamma$. Let $T$ be a solution. By $[8]$ we see $T=\partial E$ for some open set $E \subset \dot{B}^{n+1}$. The regularity theorem, see [11], for least area boundary implies that $A L B_{\rho}\left(x_{0}\right) \subset E \subset$ $B L B_{p}\left(x_{0}\right)$. Let $\tau_{a}: x \rightarrow x+a$ be a translation with $|a|$ small, then $\tau_{a} \# T$ is a solution of the oriented plateau problem with boundary $\tau_{a} \# \Gamma$. By our choice of $\Gamma$, and a similar argument to that above, we have

$$
A L B_{\rho}\left(x_{0}\right) \subset \tau_{a} \# E L B_{\rho}\left(x_{0}\right) \subset B L B_{\rho}\left(x_{0}\right), \quad \text { for all a } \mathbb{R}^{n+1}
$$

with $|a|$ small.

In particular, $x_{0} \in \operatorname{spt}\left(\tau_{a} \# T\right)$, that is, $\tau_{a}^{-1}\left(x_{0}\right) \in \operatorname{spt}(T)$, for all $a \in \mathbb{E}^{n+1}$ with $|a|$ small. This is impossible.

Now we would like to show that one-sided area minimality and onesided approximability by a non-positive mean curvature, smooth hypersurface are equivalent for minimal hypercones.

PROPOSITION 4. Let $\mathbb{L}$ and $E_{+}$be as in Theorem 1 . If $\mathbb{L}$ is not area minimizing in $\vec{E}_{+}$, then there is an $\varepsilon_{0}=\varepsilon_{0}(\mathbb{C})>0$ such that any smooth embedded hypersurface $M$ with non-positive mean curvature in $\bar{E}_{+}$, with boundary $\partial M$ outside $B_{1}$, satisfies spt $(M) \cap B_{\varepsilon_{0}}=\phi$.

Proof. Let $R$ be an oriented boundary which solves the obstacle problem:

(*) $\operatorname{Min}\left(\operatorname{Mass}(Q): Q \in I_{n}\left(\mathbb{R}^{n+1}\right)\right.$ with spt $(Q) \subseteq \bar{E}_{+}$and $\partial Q=\mathbb{E} L \mathbb{S}^{n}$ \} 
The existence of such an $R$ and its boundary regularity were shown in $[8]$.

We claim spt $(M) \cap \operatorname{spt}(R)=\phi$. This can be verified as follows. We let $\lambda_{0}>0$ be such that $\lambda_{0}=\sup \left[\lambda \in(0,1], \operatorname{spt}\left(C_{t}^{*}\right) \cap \operatorname{spt}(M)=\phi\right.$ for all $t \leq \lambda\}$, where $C_{t}^{*}=\mu_{t} \# R+\left(\mathbb{L}_{1}-C_{t}\right)$. The existence of such $\lambda_{0}$ is obvious. If $\lambda_{0}<1$, then we see $\operatorname{spt}\left(\mu_{\lambda_{0}} \# R\right) \cap \operatorname{spt}(M) \neq \phi$ and spt $\left(\mu_{\lambda_{0}} \# R\right)$ lies in one side of spt $(M)$, this contradicts Lemma 3.

Now take $\varepsilon_{0}=\sup \left\{\varepsilon>0, B_{\varepsilon}(0) \cap \operatorname{spt}(R)=\phi\right\}$, and $\varepsilon_{0}>0$ by the Maximum-Principle.

Remark. (1) In Proposition 4, $\mathbb{C}$ can be replaced by any stationary oriented boundary.

(2) Proposition 4 also shows that locally area minimality is essential in proofs of the classical "Bridge-Principle," as in [9], [6]. see also [2].

Proof of Theorem 1. Part (i) is contained in Theorem 2 which will be proved in the next section.

We first consider the case where $\mathbb{L}$ is area minimizing in $\bar{E}_{+}$. Then we have $S_{\lambda}=\mu_{\lambda} \# S_{+}$is an area minimizing hypersurface in $E_{+} \cap \mathbb{L}_{\varepsilon / 2}$ for a suitable small $\lambda>0$. For $\lambda$ small, one can assume $\partial\left(S_{\lambda} L B_{2}(0)\right)$ is $c^{k+1}$ close to $\mathbb{L L S _ { 2 } ^ { n }}$, and since $S_{\lambda} L B_{2}(0)$ is strictly stable, the implicit function theorem applies, see [9], to yield the existence of smooth embedded hypersurfaces $M_{\varepsilon}^{+}, M_{\varepsilon}^{-}$lying in $E_{\varepsilon} \cap E_{+} \cap B_{2}(0)$ with constant (small) positive and negative mean curvature respectively. Moreover $\partial M_{E}^{+}=\partial M_{E}^{-}=\partial\left(S_{\lambda} L B_{2}(0)\right)$. This proves (iii).

To show (ii), we consider, for small $\delta>0$, the obstacle problems $\min \left\{M(Q): Q \in I_{n}\left(\mathbb{R}^{n+1}\right)\right.$ with spt $(Q) \subseteq \bar{E}_{+}$and $\left.\partial Q=\Gamma_{\delta}\right\}$, where $\Gamma_{\delta}=\left\{x+\delta v_{c}(x): x \in \partial \mathbb{E}_{1}\right\}$. By [5, section 5] and [8] we obtain solutions $T_{\delta}$ to the above problems with sing $\left(T_{\delta}\right)=\phi$, for all $\delta>0$ sufficiently small. It is at this step we need the assumption $n=7$. 
Next we apply perturbation results of [12] to obtain $M_{\delta}$ which are smooth embedded hypersurfaces in $E_{+}$with positive mean curvature and $\partial M_{\delta}=\partial T_{\delta}=\Gamma_{\delta}$. We also observe that $\mathbb{L}_{1,2 / \varepsilon}=\mathbb{L}\left(B_{2 / \varepsilon}-B_{1}\right)$ is strictly stable, hence for all sufficiently small $\delta>0$, one can find a smooth embedded hypersurface $\tilde{M}_{\delta}$ in $E_{+}$with positive mean-curvature and (which is close to $\left.\Gamma_{1,2 / \varepsilon}\right)$ such that $\partial \tilde{M}_{\delta}=-\Gamma_{\delta}+\tilde{\Gamma}_{\delta}, \tilde{\Gamma}_{\delta}=\left\{x+\delta v_{c}(x)\right.$ : $\left.x \in \partial \mathbb{L}_{2 / \varepsilon}\right\}$.

Now we claim the angle made by $M_{\delta}$ and $\tilde{M}_{\delta}$ along $\Gamma_{\delta}$ in the positive mean curvature direction is strictly less than $\pi$ for all small $\delta>0$. This can be verified by a contradiction argument. Since as $\delta \rightarrow 0^{+}, \tilde{M}_{\delta} \rightarrow \mathbb{L}_{1,2 / \varepsilon}$ and $M_{\delta} \rightarrow R$ a one-sided area minimizing integral current with boundary $\Gamma_{0}=\partial \mathbb{l}_{1}$ (by taking a sub-sequence if necessary). By the boundary regularity of $R$, see [8], and the Hopf-boundary point lemma, we see spt $(R)$ and spt $(E)$ intersect transversely along $\Gamma_{0}$. Therefore we can smooth the corner made by $M_{\delta}$ and $\tilde{M}_{\delta}$ to obtain $M$ which is a smooth embedded hypersurface with positive mean curvature in $E_{+}$. Finally we let $M_{\varepsilon}=\mu_{\varepsilon} \# M$ to yield (ii).

\section{Global approximation}

Let $\mathbb{C}$ be a regular minimal hypercone, by [5], the main result of [3] can be generalized to the case of a faster decay solution at infinity, that is, for a $C^{\alpha}$-function $f$ on $\mathbb{C}$ which decays at $\infty$ at a sufficiently faster rate, and with $\|f\|_{C^{\alpha}}$ small, then one can find a solution of $M_{c} u=f$ on $\mathbb{L}_{1, \infty}$, with $\|u\|_{C^{2, \alpha}}$ small, and $u$ decays to $\mathbb{l}$ at infinity at a sufficiently faster rate.

- A regular minimal hypercone $\mathbb{C}$ is called one-sided strictly area minimizing in $\bar{E}_{+}$if there is $\theta>0$ such that

$$
M\left(\mathbb{L}_{1}\right) \leq M(S)-\theta \varepsilon^{n}
$$

whenever $1>\varepsilon>0$ and $S$ is an integral current with spt $(S) \subseteq \bar{E}_{+} \sim B_{\varepsilon}$ 
and $\partial S=\partial C_{1}$, see $[7],[5]$.

As for strictly area minimizing hypercones, we have the following consequence of [5, Theorem 3.2] which gives various characterizations of one-sided strictly area minimizing hypercones.

LEMMA 5. Let $\mathbb{L}, E_{+}, S_{+}, U_{+}$be as in Section II, then the folzowing are equivalent:

(i) $\mathbb{C}$ is strictly area minimizing in $\bar{E}_{+}$.

(ii) $U_{+}$has slower decay at infinity. That is

$\lim _{|x| \rightarrow \infty} U_{+}(x) /|x|^{-\gamma} \equiv c>0$, in the case $\gamma_{+}>\gamma_{-}$

$\lim _{|x| \rightarrow \infty} U_{+}(x) /(\log |x|) \cdot|x|^{-(n-2) / 2}=c>0$, in the case $\gamma_{+}=\gamma_{-}=\frac{n-2}{2}$.

(iii) For any non-negative $g_{+} \in C^{1}\left(S_{+}\right)$which decays at infinity faster than $|x|^{-\gamma_{+}^{-3}}$, there exists a positive solution $W_{+}$of $L_{S_{+}} W_{+}=-g_{+}$

(iv) For any non-negative $g_{+} \in C^{1}\left(S_{+}\right)$which decays at $\infty$ faster than $|x|^{-\gamma_{+}^{-3}}$, there exists on $\varepsilon_{0}>0$ such that for every $\varepsilon \in\left(0, \varepsilon_{0}{ }^{\prime}\right.$, there is a positive $C^{2}\left(S_{+}\right)$solution of $M_{S_{+}}{ }^{E}{ }_{+}^{\varepsilon}=-\varepsilon g_{+}$.

Remark. (1) The proof of the above Lemma can be found in [5, Section 3], except that (iii) and (iv) should be slightly modified. The reason why we can allow the condition $" g_{+}$decays sufficiently faster at $\infty$ " instead of "compact support" is that $\phi=\left(\gamma^{-\gamma_{+}}-\gamma^{-\gamma_{+}}\right)^{-\alpha_{\phi_{1}}}$ satisfies $L_{S_{+}} \phi \leq-C_{0} \gamma^{-\gamma_{+}-2-\alpha}$ on $S_{+}-B_{R_{0}}$ where $\alpha \in(0,1), R_{0}, C_{0}$ are two positive constants depending only on $\alpha$ and the cone $\mathbb{L}$. For the details the reader should see [5, section 3$]$.

(2) By the same proof as for (iv), one can show there is a negative $C^{2}\left(S_{+}\right)$solution of $M_{S_{+}} W_{-}^{\varepsilon}=\varepsilon g_{+}$, for $\varepsilon \in\left(0, \varepsilon_{0}\right)$.

Now we can prove Theorem 2 . 
Proof. of Theorem 2. Part (i): For given $\varepsilon>0$, we let $u$ be a $c^{2, \alpha}$ solution of $M_{\mathbb{L}} u=f>0$ in $\mathbb{L}_{1, \infty}$, and $\left.u\right|_{\partial \mathbb{L}_{1}}=0$, such that $\|u\|_{c^{2, \alpha}} \leq \varepsilon$, where $f$ is a smooth positive function defined on the cone $\mathbb{L}$ which decays faster at $\infty$. Hence $\operatorname{graph}_{\mathbb{C}} U=\left\{x+u(x) v_{c}(x): x \in \mathbb{L}_{1},{ }_{\infty}\right\}$ is a smooth embedded hypersurface with boundary and positive mean-curvature.

Now let $R$ be as in the Proof of Proposition 4, so that $\partial R=\partial \mathbb{C}_{1}$ and $R$ is area-minimizing in $E_{+}$. suppose spt $(R)$ is a smooth embedded hypersurface with boundary (this will be the case if $n \leq 6$ ), then we apply the perturbation of the theorem of [12] to obtain a smooth embedded hypersurface $M$ of positive mean curvature in $E_{+}$such that $\partial M=\partial \mathbb{L}_{1}$ and $M$ is sufficiently close to $R$. As in the Proof of Theorem 1, Part (ii), we see that the corner angle made by $M$ and graph $\mathbb{U}^{U}$ is strictly less than $\pi$ if $\varepsilon$ is sufficiently small. Therefore we can smooth the corner to obtain a new surface $\tilde{M}$ with positive mean curvature. Let $M_{\varepsilon}=U_{\varepsilon} \# \tilde{M}$, then $M_{\varepsilon}$ satisfies the conclusion (i). Now suppose sing $(R) \neq \phi$, then we consider the obstacle problem:

$$
\begin{aligned}
\min \{M(Q) & : Q \in I_{n}\left(\mathbb{R}^{n+1}\right) \text { with } \operatorname{spt}(Q) \subset \mathbb{L}_{\varepsilon} \cup \bar{E}_{+} \text {and } \partial Q \\
& \left.=\left\{x-\delta v_{o}(x): x \in \partial \mathbb{L}_{2}\right\}\right\}
\end{aligned}
$$

where $\delta \in(0, \varepsilon)$. Let $T_{\delta}$ be a solution of above problem, then $T_{\delta} \rightarrow R^{*}$ as $\delta \rightarrow 0^{+}$, for some $R^{*}$ a solution to the corresponding problem with $\delta=0$. Then, by $[8]$, we see the uniformly boundary regularity of $T_{\delta}$ 's for $\delta$ positive and small, and by $[5$, section 5] we can assume sing $\left(T_{\delta_{m}}\right)=\phi$ for some $\delta_{m} \rightarrow 0^{+}$. Since we can choose graph $U$ as close to $\mathbb{C}_{1}, \infty$ as we want, and spt $\left(R^{*}\right)$ is smooth near $\partial \mathbb{C}_{2}$ and intersects $\mathbb{L}$ transversely slong $\partial \mathscr{L}_{2}$, we can choose a proper $\delta_{m}>0$ and graph $U$, so that spt $\left(T_{\delta_{m}}\right)$ intersects graph $\frac{\mathbb{L}}{d}$ transversely along some submanifold which is close to $\partial \mathbb{E}_{2}$. Moreover we can smooth the 
corner to obtain a new surface of positive mean-survature $\tilde{M}$ as before, and finally we set $M_{\varepsilon}=\mu_{\varepsilon} \# \tilde{M}$. This finishes the Proof of Part (i). Part (ii): Follows directly from Lemma 5 and Remark 2 following Lemma 5.

\section{General cases}

In this final section, we would like to make a few remarks about one-side local approximation of an area minimizing oriented boundary or area minimizing boundary with contained volume by smooth properly embedded hypersurfaces of positive mean curvature.

Let $N$ be a complete $(n+1)$-dimensional smooth Riemannian manifold, and let $E, U, V$ be open subsets of $N$ with $E \subset U, V \subset \subset U$, and let $T=\partial E L U$ be an oriented boundary of least area in $U$ with spt $(T)=$ $\partial E\llcorner U$. We also assume that $\xi \in$ sing $(T)$ implies that there is a regular tangent cone $C(\xi)$ at $\xi$ for $T$. Also we fix a $C^{2}$-comain $W \subset V$ such that spt $T \cap \partial W \subset \operatorname{reg}(T)$ and the intersection is transverse, with $\Gamma_{0}=\partial(T L W)$.

Then we have the following.

PROPOSITION 6. For any $\varepsilon>0$ there is a smooth, properly embedded hypersurface $M_{\varepsilon}^{+}$(respectively $M_{\varepsilon}^{-}$), in an $\varepsilon$-neighbourhood of spt $(T)$, of positive (respectively negative) mean curvature, and such that $\partial M_{\varepsilon}^{ \pm}=\Gamma=\phi \# \Gamma_{0}$ for some $\phi \in C^{2}\left(\Gamma_{0}\right)$ with $\left|\phi-i_{\Gamma_{0}}\right|_{C^{2}}<\varepsilon$. Moreover $M_{\varepsilon}^{ \pm} \subset E$.

Proof. This is an easy consequence of a result of [5, section 5] and the implicit function theorem.

PROPOSITION 7. For $n \leq 7$, let $\Omega \subset N$ be such that a $\Omega$ has least area for the contained volume. Then $\partial \Omega$ can be approximated by a sequence of smooth embedded hypersurfaces $\left\{M_{m}\right\}_{m=1}^{\infty}$, lie inside $\bar{\Omega}$, and each $M_{m}$ has positive mean curvature.

Proof. By [4] and [11], the conclusing is trivial when $n \leq 6$. For the case $n=7, \partial \Omega$ has at most isolated singularities (hence 


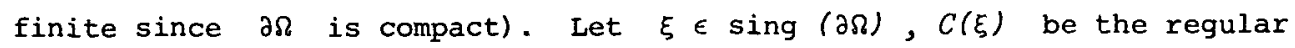
minimizing tangent cone of $\partial \Omega$ at $\xi$, see [10]. After a suitable scaling, we can assume that $\partial \Omega L B_{1}(\xi)$ sufficiently close to $C(\xi) L B_{1}(\xi)$, and that $\Gamma=\partial \Omega L \Phi_{1}(\xi)$ is a smooth 6-dimensional submanifold of $\Phi_{1}(\xi)=\partial B_{1}(\xi)$. One solves the oriented plateau probiem with boundary $\Gamma$ in $\bar{\Omega} \cap B_{1}(\xi)$, the resulting solutions $M$ will be close to $C(\xi)$ since $\Gamma$ is close to $\partial \mathbb{L}_{1}(\xi)$. By the maximum-principle (see Lemma 1 also), spt $(M) \cap \partial \Omega=\Gamma$ and the intersection is transverse by the Hopfboundary point lemma. We can assume sing $(M)=\phi$ otherwise replace $M$ by a solution of the oriented plateau problem with boundary $\Gamma_{\varepsilon}=$ $\partial \Omega L \Phi_{1+\varepsilon}(\xi)$ for $\varepsilon>0$ small, this follows from [5, Section 5].

Since $M$ is smooth, one can apply the implicit function theorems as in [12] to obtain a new hypersurface $\bar{M} \bar{\Omega}$ with positive mean curvature and $\partial \bar{M}=\Gamma, \bar{M} \cap \partial \Omega=\Gamma$, and the intersection is transverse. By the same argument as before we can smooth corners to find resulting hypersurfaces properly embedded in $\bar{\Omega}$, of positive mean curvature.

\section{References}

[1] Geometric measure theory and the calculus of variations. (Proc. Sympos. Pure Math. 44, W.K. Allard and F.J. Almgren Jr., Eds.., American Mathematical Society, Providence, Rhode Island, 1986).

[2] F.J. Almgren, B. Solomon, "How to connect minimal surfaces by bridges", Abstracts Ams. Math. Soc. (1980), 775-B7.

[3] L. Caffarelli, R. Hardt, L. Simon, "Minimal surfaces with isolated singularities", Monuscripta Math. 48 (1984), 1-18.

[4] E. Gouzalez, U. Massari, I. Tamanini, "On the regularity of bodies of sets minimizing perimeter with a volume constraint", Indiana Univ. Math. J. 32 (1983), 25-37.

[5] R. Hardt, L. Simon, "Area minimizing hypersurfaces with isolated singularities", J. Reine Angew. Math. 362 (1985), 102-129.

[6] B. Lawson, M.L. Michelsohn, "Embedding and surrounding with positive mean curvature", Inv Math. 77 (1984), 399-419.

[7] F.H. Lin, "Minimality and stability of minimal hypersurfaces in $\mathbb{B}^{n+1}$ "., Bull. Austral. Math. Soc. (Paper \#6546). 
[8] F.H. Lin, Regularity for a class of parconetric obstacle problem, Ph.D. Thesis (University of Minnesota, Minneapolis, 1985).

[9] W. Meeks, S.T. Yau, "The existence of embedded minimal surfaces and the porblem of uniqueness", Math. 2. 179 (1982), 151-168.

[10] L. Simon, "Isolated singularities of extrema of geometric variational problems", C.M.A. Research Report, (Australian National University, 1984).

[11] L. Simon, Lectures on Geometric Measure Theory, (Centre for Mathematical Analysis, Australian National University, 1983),

[12] F. Tomi, "A perturbation theorem for surfaces of constant mean curvature", Math. Z. 141 (1975), 253-263.

Courant Institute of Mathematical Sciences

New York University

New York, New York 10012 